\title{
Fratricide effect on ELTs
}

\author{
Damien Gratadour $^{1, a}$, Eric Gendron ${ }^{1}$, Gerard Rousset ${ }^{1}$, and Francois Rigaut ${ }^{2}$ \\ 1 Laboratoire d'Etudes Spatiales et d'Instrumentation en Astrophysique (LESIA), Observatoire de \\ Paris, CNRS, UPMC, Universite Paris Diderot, 5 places Jules Janssen, 92195 Meudon France ${ }^{c}$ \\ 2 Gemini Observatory Southern Operation Center, c/o AURA, Casilla 603, La Serena, Chile
}

\begin{abstract}
In this paper, we present the results of our study of the so-called fratricide effect, i.e. the increased background observed in a number of a wavefront sensor's sub-apertures, due to the scattering of laser photons on dust or clouds particles or air molecules located along a laser beam. Using the formalism developed by the LIDAR community, we computed the background levels to be expected on various kinds of adaptive optics systems to be installed eventually on the E-ELT (42 m). In the case of pure Rayleigh backscattering, the induced background, expected to be constant (as it mainly depends on the air molecules density), is not a show stopper. We however stress that in the presence of sub-visible clouds or dust particles along the laser beam, the significant amount of induced background, which is likely to be variable, could potentially affect the performance of AO systems.
\end{abstract}

\section{Introduction}

The fratricide effect is due to the scattering of laser photons on dust or clouds particles (Mie scattering) or air molecules (Rayleigh scattering) located along a laser beam projected to the sky. It appears in AO systems using laser guide stars, when photons of one or several laser beams are scattered along the line of sight of some of a WFS sub apertures. Mie and Rayleigh scattering are essentially described by the same equations and, to the first order, it can be considered that they only differ by their scattering cross-section.

While the Rayleigh scattering of laser photons in the atmosphere is a well-characterized phenomena, thanks to the many LIDAR studies ; the parameters of Mie scattering are highly dependant on weather (cloud density) and day-to-day site conditions (dust). We thus concentrated on Rayleigh scattering assuming that our results could be easily extended to the case of a mixture of Rayleigh and Mie scattering provided the knowledge of the weather and site conditions. We note however that when adding Mie scattering, things (i.e. residual wavefront error) will obviously get worse.

As a first order approximation, it can be assumed that the laser beams themselves, viewed from the WFS, are completely out of focus, so the photon return from Rayleigh backscattering can be considered as an inhomogeneous background over the area of one sub-aperture.

We used the molecules profile provided by ESO in (document E-SPE-ESO-276-0206) and the Rayleigh backscatter cross section widely used in the LIDAR community : $5.45^{*}(550 / \lambda)^{4}$ in units of $10^{-32} \mathrm{~m}^{2} \cdot \mathrm{sr}^{-1}$. Each laser beam is sampled in altitude in the same fashion as was the molecules profile. The contribution of each slab of laser beam on the WFS is modelled as a Gaussian normalized to the total number of photons scattered by that slab and with the proper position angle with respect to the sub aperture. The FWHM of this Gaussian is the quadratic sum of the laser beam diameter, the seeing and the blur caused by the beam defocus.

\section{Rayleigh background distribution}

The Rayleigh background distribution on the sub apertures of a LGS AO system (Shack-Hartmann WFS with $84 \times 84$ sub apertures) on the E-ELT (diameter $=42 \mathrm{~m}$, central obstruction $=0.3$ ) can be

\footnotetext{
${ }^{c}$ Groupement d'Interet Scientifique PHASE (Partenariat Haute resolution Angulaire Sol Espace) between ONERA, Observatoire de Paris, CNRS and Universite Paris Diderot

a e-mail: damien.gratadour@obspm.fr
}

This is an Open Access article distributed under the terms of the Creative Commons Attribution-Noncommercial License, which permits unrestricted use, distribution, and reproduction in any noncommercial medium, provided the original work is properly cited. 

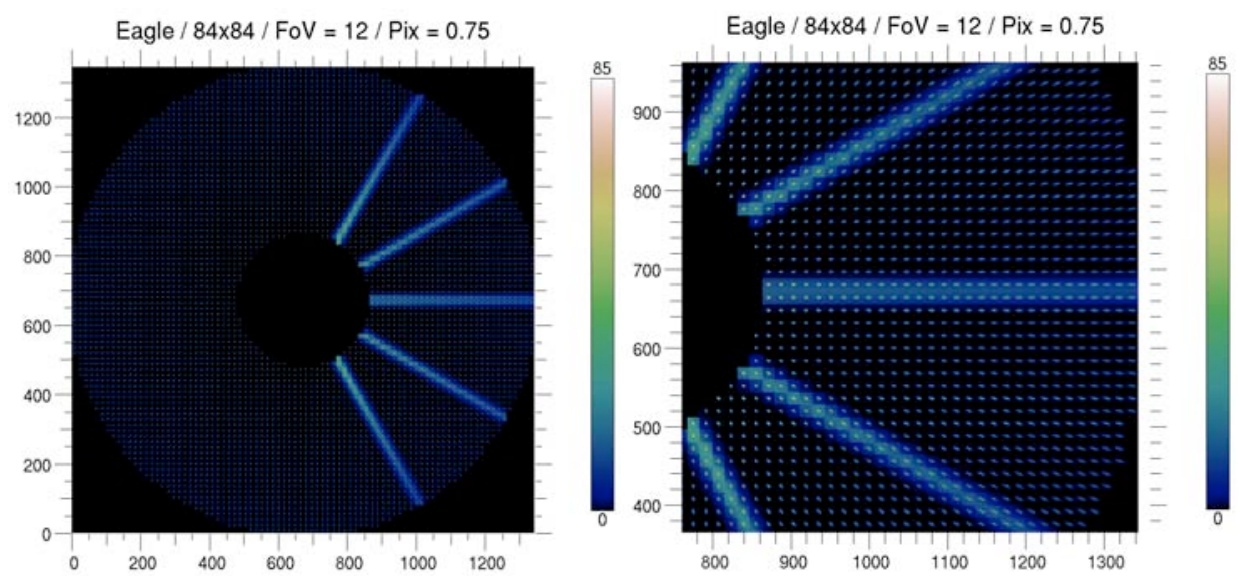

Eagle - Rayeigh photons / arcsec2
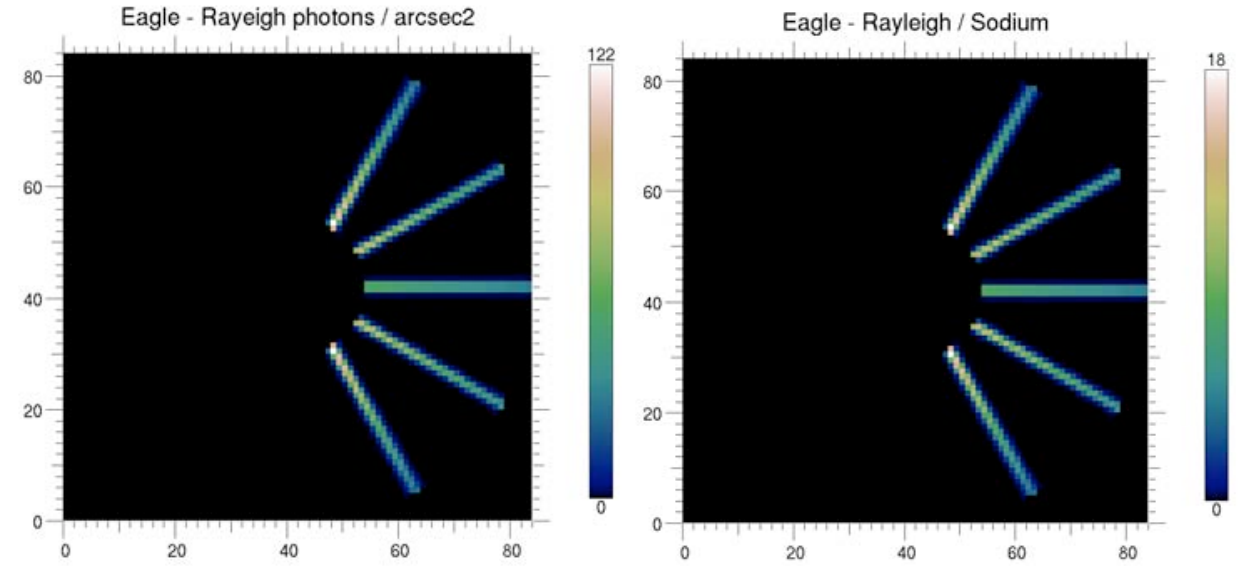

Fig. 1. EAGLE case (LGS constellation radius $=3.6 \mathrm{arcmin}$ ), from left to right, top to bottom: Rayleigh background + Na spot images of a $84 \times 84$ SH system ; zoom on the affected sub apertures ; number of Rayleigh photons per $\operatorname{arcsec}^{2}$ and per $2 \mathrm{~ms}$; Rayleigh over Na ratio for each sub aperture.

computed with available computers. A modified version of some parts of the YAO code written by Francois Rigaut ([1] ) was used to do these calculations.

We studied two kinds of configurations for a 6 LGS AO system on the E-ELT:

- a LGS constellation with a 3.6 arcmin radius, i.e. EAGLE case

- a LGS constellation with a 30 arcsec radius, i.e. LTAO case. We chose a laser power of $10 \mathrm{~W}$ per LGS in both cases.

The results are presented in figures 1 and 2 respectively, in which we show the Rayleigh background + Na spot images, the number of Rayleigh photons per $\operatorname{arcsec}^{2}$ and per $2 \mathrm{~ms}(500 \mathrm{~Hz})$ and the Rayleigh over Na ratio for each sub aperture of a $84 \times 84$ SH system. In both cases, there were 16x16 pixels of 0.75 arcsec in each sub aperture leading to a field of view of 12 arcsec.

The Na photon return was computed assuming an empirical parameter to convert from laser power to fluorescence photon return. This parameter leads to an average of 250 photons detected per $50 \mathrm{~cm}^{2}$ sub apertures and per ms with a $10 \mathrm{~W}$ laser. This is what is commonly observed on a system like Altair on Gemini North during the high Na content season. The resulting photon return can then be distributed along either a Gaussian or any other profile to account for Na layer distribution (i.e. spot elongation and shape). We used a Gaussian Na layer distribution with a $10 \mathrm{~km} \mathrm{FWHM}$ and located at $90 \mathrm{~km}$. 


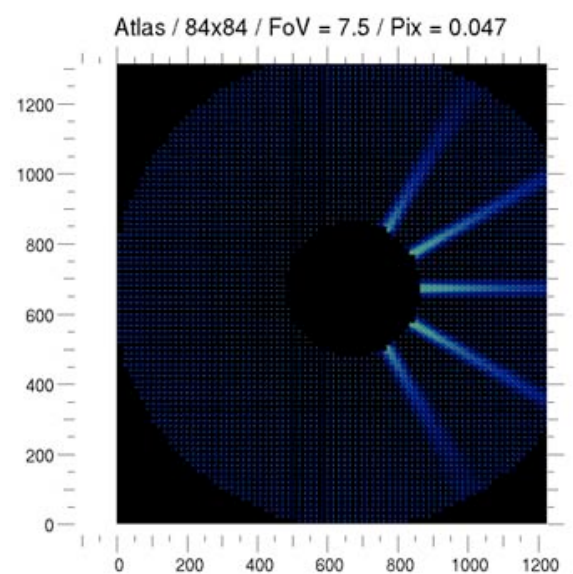

Atlas - Rayleigh photons / arcsec2

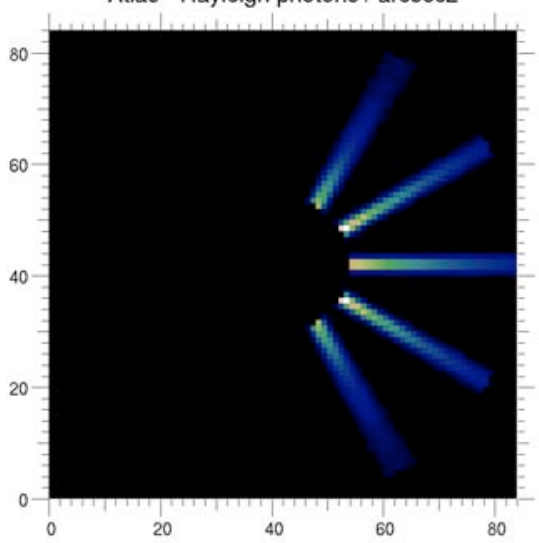

Atlas $/ 84 \times 84 /$ FoV $=7.5 /$ Pix $=0.047$

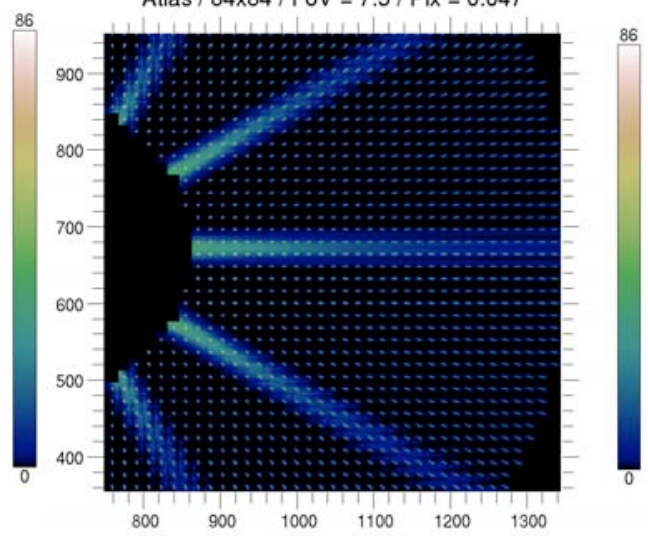

Atlas - Rayleigh / Sodium

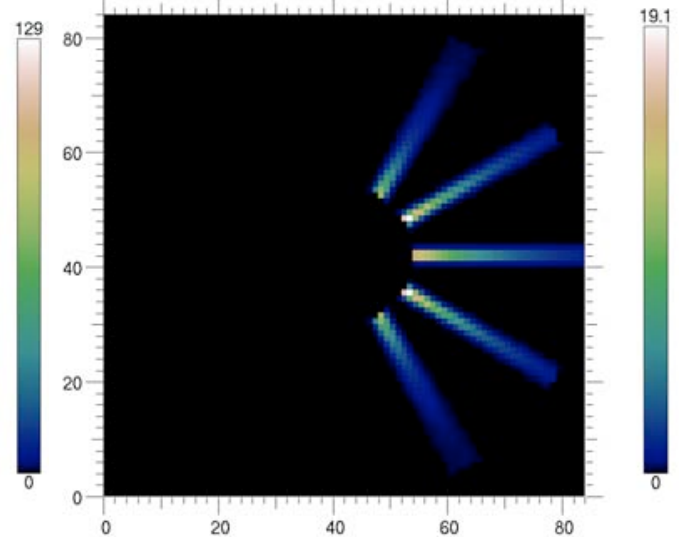

Fig. 2. LTAO case (LGS constellation radius $=30$ arcsec), from left to right, top to bottom: Rayleigh background + Na spot images of a 84x84 SH system ; zoom on the affected sub apertures ; number of Rayleigh photons per $\operatorname{arcsec}^{2}$ and per $2 \mathrm{~ms}$; Rayleigh over Na ratio for each sub aperture.

As we study the case of central launching the Rayleigh background distribution for the other WFS will be the same with a $60 \mathrm{deg}$ rotation. The spot elongation distribution will remain the same.

While the maximum number of Rayleigh photons are similar in both cases, the decrease with the distance to the centre of the pupil is slower in the EAGLE case. This can be easily understood using geometrical considerations depicted in Figure 3 in which each laser beam is represented by an oblique line (with the proper angle).

On this figure, the grey area delimitates the portion of each laser beam from which photons will be scattered along the line of sight of at least one sub aperture of the 6th WFS. The photons from the lower part of this portion of laser beam will end up in the central sub apertures while the photons from the upper part will end up in the peripheral sub apertures. Taking into account the 0.3 central obstruction, the central sub apertures in the EAGLE case will see a portion of the beam located at about $5 \mathrm{~km}$ and the peripheral sub apertures a portion of the laser beam located at about $17 \mathrm{~km}$. In the LTAO case the lower portion of the beam intercepted by the FoV of the central sub apertures is located at $8 \mathrm{~km}$ and the upper portion is located at about $45 \mathrm{~km}$.

Moreover, the volume of the beam portion intercepted by the peripheral sub apertures FoV (hence located at higher altitudes) is about 5 times larger than the volume of the beam portion intercepted by the central sub apertures FoV in the EAGLE case while there is a factor of only 1.5 in the LTAO case. If we compare this rough estimates to the molecules profile, we find a good agreement with what is 


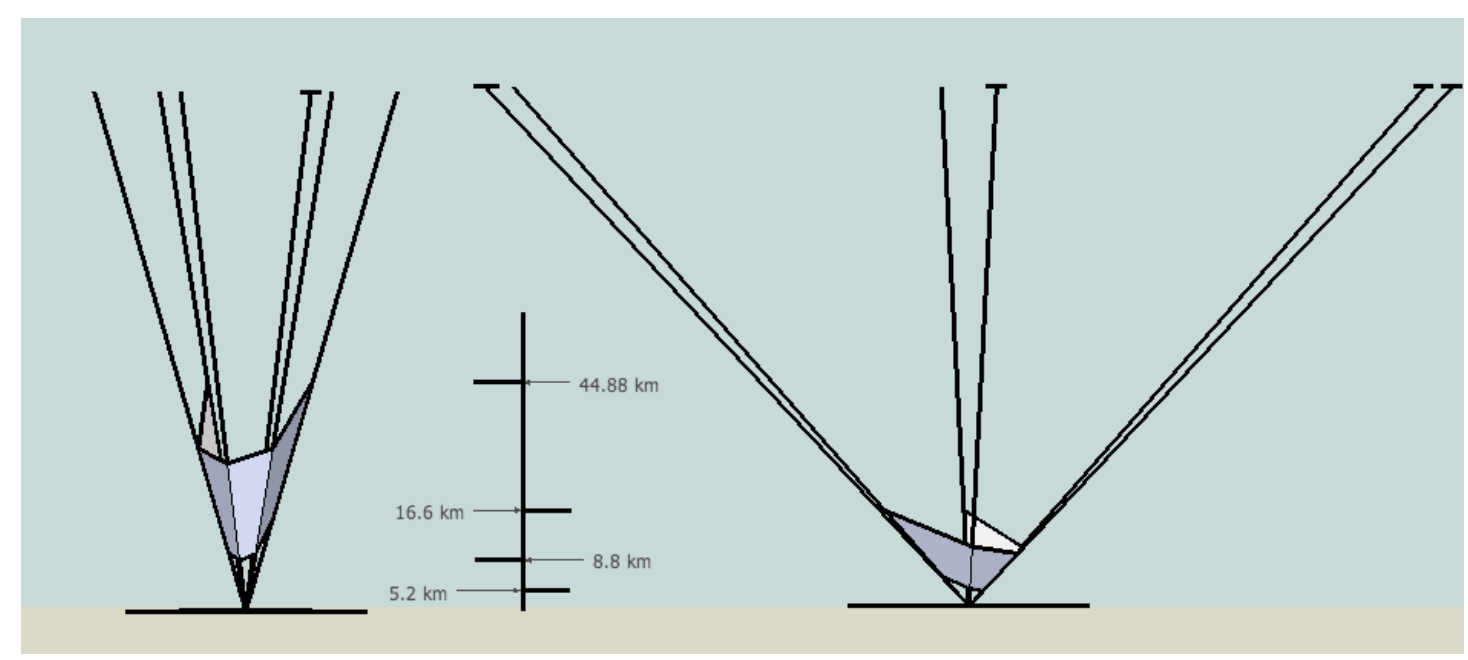

Fig. 3. Sketch representing the two launch configurations (left: LTAO, right: EAGLE). Each oblique line is a laser beam. The grey area delimitates the portion of the laser beams from which photons will be scattered along the line of sight of at least one sub aperture of the 6th WFS.

observed in figure 1 and 2. Indeed, the number of molecules is 4 times larger at $5 \mathrm{~km}$ than at $15 \mathrm{~km}$. This explains the very slow decrease of the Rayleigh background level across the pupil in the EAGLE case. In the LTAO case, there is a factor of about 130 in molecules density between 8 and $40 \mathrm{~km}$ which explains the fast decrease of the Rayleigh background level.

\section{Error induced on the wavefront sensor}

This geometric effect will translate into enhanced wavefront estimation error as shown on the left figure. Assuming a SH WFS using a Weighted Center of Gravity for slope estimation, analytical formulas can be used to derive RMS OPD error as shown in [3]. Fratricide effect can be taken into account using this formalism assuming a constant Rayleogh background across one sub-aperture which is then assimilated to a uniform background.This figure shows the relatively limited impact of fratricide effect which moreover applies only to the sub-apertures located along one LGS beam footprint.

In order to estimate the overall residual phase error induced by this effect, we need to use a tomographic reconstructor. Available computers do not allow yet to compute such a reconstructor for a 84x84 SH system on the E-ELT. We note however that :

- The Rayleigh / Na ratio in the case of a $16 \times 16 \mathrm{SH}$ on a $8 \mathrm{~m}$ telescope is of about 12 which is lower than what we found here but not by an order of magnitude (the main difference between the 2 cases is the FoV of the sub apertures)

- The proportion of sub apertures affected by Rayleigh background decreases with the telescope diameter. Hence assuming comparable Rayleigh / Na ratio in the affected sub apertures the impact of fratricide effect will be reduced as we increase the telescope diameter, thus the number of sub apertures.

From these two observations, we can conclude that the results on an intermediate case would give us a good estimate of the final impact of fratricide effect on the residual wavefront. Simulations on such a downscaled systems have been performed and are descibed in details in [3].

They show that while central and edge launching are leading to comparable performance without fratricide effect, adding the latter introduce an additional $40 \mathrm{~nm}$ to the error budget of the central launching which leads to a total WFE of $78 \mathrm{~nm}$, slightly larger than the edge launching case. We stress however that this is a rather small contribution to the global error budget. 


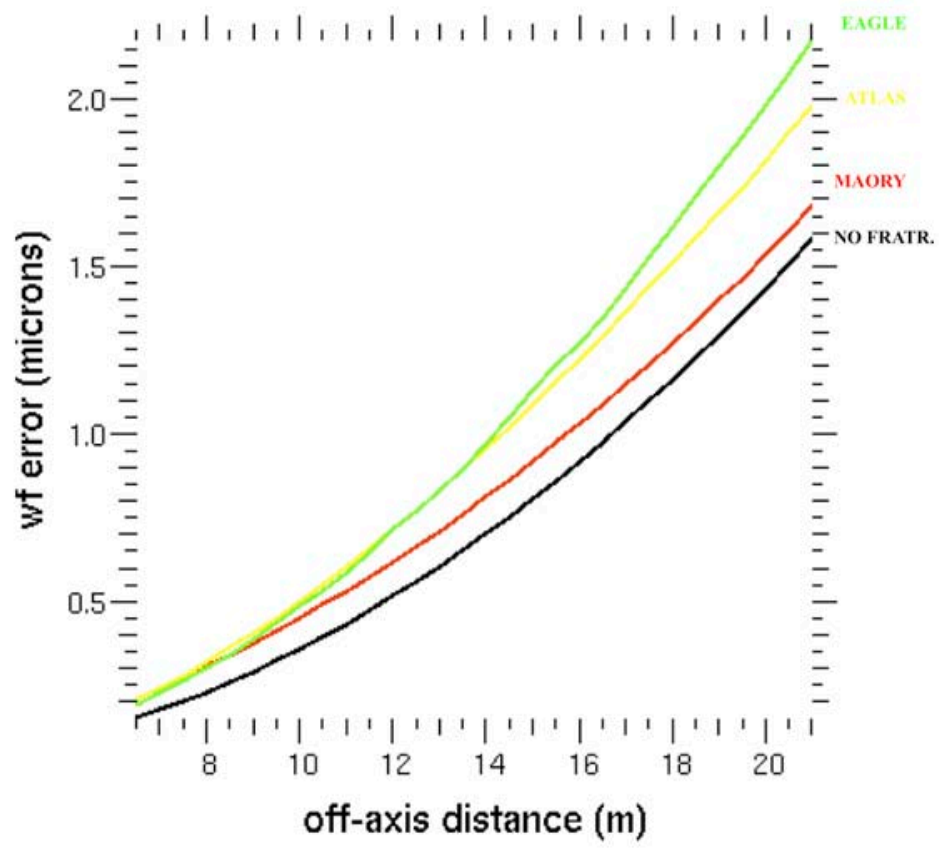

Fig. 4. Wavefront error induced on the WFS sub-apertures with respect to their distance to the laser launch position, without (plot labelled no fratr.) and with fratricide effect in various conditions (i.e. various LGS asterism opening angle).

Finally, we can note that this rather small contribution can probably be explained by the fact that the sub apertures in which the ratio Rayleigh / $\mathrm{Na}$ is the highest are the central ones, i.e. where the spots are less elongated. This is especially true in the LTAO case.

\section{Conclusion}

Fratricide effect due to pure Rayleigh backscattering is not a show stopper for multiple LGS AO systems on ELTs. However, a variable background induced for instance by Mie scattering on dust particles could be an important issue but has not been studied yet, due to the lack of data.

\section{References}

1. Francois Rigaut, http://www.maumae.net/yao/aosimul.html

2. Nicolle et al, Optics Letters (Vol 29, Issue 23, 2004) pp. 2743-2745

3. Robert, Conan, Gratadour, Fusco, Petit, Sauvage, Muller, this conference 